\title{
QUALIDADE MICROBIOLÓGICA DE SUSHI COMERCIALIZADO NA CIDADE DE ERECHIM - RIO GRANDE DO SUL
}

\author{
Microbiological quality of sushi sold in the city of Erechim - \\ Rio Grande do Sul state
}

Bruna Chmiel ${ }^{1}$; Cilda Piccoli²; Geciane Toniazzo Backes ${ }^{3}$; Roseana Baggio Spinelli ${ }^{4}$

\begin{abstract}
${ }^{1}$ Acadêmica do Curso de Nutrição da Universidade Regional Integrada do Alto Uruguai e das Missões, URI Erechim.E-mail: bruna_chmiel@outlook.com

${ }^{2}$ Nutricionista, Docente do Curso de Nutrição da Universidade Regional Integrada do Alto Uruguai e das Missões, URI Erechim, Mestra em Engenharia de Alimentos pela Universidade Regional Integrada do Alto Uruguai e das Missões, URI Erechim.

${ }^{3}$ Engenheira de Alimentos, Docente do Curso de Nutrição da Universidade Regional Integrada do Alto Uruguai e das Missões, URI Erechim, Doutora em Ciências pela Universidade Federal do Rio de Janeiro, UFRJ - Rio de Janeiro.

${ }^{4}$ Nutricionista, Docente do Curso de Nutrição da Universidade Regional Integrada do Alto Uruguai e das Missões, URI Erechim, RS, Mestre em Gerontologia Biomédica (PUCRS).
\end{abstract}

RESUMO: O consumo de sushi, prato típico japonês, preparado a partir de peixe cru, tornou-se muito popular e consumido em muitos países. Entretanto, ele oferece um risco grande à saúde por não ser submetido ao processo de cocção, podendo ocasionar uma contaminação por microrganismos patogênicos. O objetivo dessa pesquisa foi avaliar as condições microbiológicas de sushi comercializado na cidade de Erechim, Rio Grande do Sul. Foram selecionados dois estabelecimentos anônimos e escolhidos por conveniência, um especializado em culinária japonesa e o outro não especializado, coletando três amostras de cada local, com uma diferença de 10 dias, no mês de janeiro de 2020. Realizaram-se análises de Escherichia coli, Staphylococcus coagulase positiva e Salmonella sp. De acordo com os resultados, o estabelecimento especializado apresentou Escherichia coli acima do permitido pela legislação em duas amostras, sendo inadequado para o consumo. O estabelecimento não especializado obteve apenas uma amostra com presença de Staphylococcus 
e as três amostras com presença de Escherichia coli, porém todas dentro do limite estabelecido pela legislação. Nenhum local apresentou presença de Salmonella. Constatou-se que se faz necessária a implantação de programas de educação em saúde, treinamentos para manipuladores de alimentos, para desta forma buscar alimentos seguros, melhorando a saúde da população.

Palavras-chave: Bactérias. Higiene dos Alimentos. Peixes.

ABSTRACT: The consumption of sushi, a typical Japanese food, prepared with raw fish, has been very popular and consumed in many countries. However, it offers a great risk to health because it is not subjected to the cooking process which may cause contamination by pathogenic microorganisms. The objective of this research was to evaluate the microbiological conditions of sushi sold in the city of Erechim, in Rio Grande do Sul state. Two anonymous establishments were selected and chosen for convenience, one specialized in Japanese cuisine and the other one non-specialized. Three samples from each place were collected, having a-10-day difference between each collection, in January 2020. Analyzes of E. coli, Estaphylococcus coagulase positive and Salmonella sp. were carried out. According to the results, the specialized establishment presented E. coli above the levels allowed by the legislation in two samples, therefore unsuitable for consumption. The non-specialized establishment obtained only one sample with the presence of Staphylococcus and three samples with the presence of E. coli, but all within the limit established by the legislation. No site showed the presence of Salmonella. It was found that it is necessary to implement health education programs, food handlers training in order to seek safe food, improving the population's health.

Keywords: Bacterias. Food Hygiene. Fish.

\section{Introdução}

O pescado é considerado um dos alimentos mais nutritivos e saudáveis para o consumo humano. É rico em proteínas de alto valor biológico, possui altas quantidades de ácidos graxos poli-insaturados Ômega 3, vitaminas lipossolúveis e contém nutrientes como fósforo, ferro, cobre, selênio e iodo (para os peixes de água salgada) (SARTORI; AMANCIO, 2012; OLIVEIRA, 2013).

Estudos demonstraram alguns benefícios para a saúde em relação ao consumo de peixes, como sendo um aliado para prevenir doenças cardíacas, depressão e acidente vascular cerebral (AVC), pois sua carne é de alto valor dietético e proteico, com pouca gordura e baixo nível de colesterol (LUNKES et al., 2018). No estudo de Mozaffarian e Wu (2012), na qual foram analisados os efeitos do consumo de PUFA ômega-3 encontrados no peixe ou no óleo de peixe, em relação aos benefícios cardiovasculares, constatou-se que em humanos, os ácidos graxos reduzem os níveis de triglicerídeos, afetam favoravelmente o enchimento diastólico cardíaco, a complacência arterial e algumas métricas de inflamação e estresse oxidativo.

O consumo de sushi, prato típico japonês, preparado a partir de peixe cru, arroz japonês e alga marinha, se tornou muito popular e consumido em outros países. No Brasil este 
alimento também faz muito sucesso, e existe uma procura grande por estabelecimentos especializados nesse tipo de culinária, que se tornou um hábito alimentar e um modismo da população pela culinária oriental (SANTOS et al., 2012).

Entretanto, condições ambientais insatisfatórias e precárias medidas higiênicosanitárias, durante a manipulação, podem ocasionar problemas à saúde dos consumidores, como uma DTA (Doença Transmitida por Alimentos) (MONTANARI et al., 2015; GUIMARÃES; SILVA; GUIMARÃES, 2016; MEDEIROS; CARVALHO; FRANCO, 2017).

Na sua preparação, além da contaminação do pescado, o contato direto com as mãos dos manipuladores, o armazenamento da matéria-prima, a higiene dos utensílios, dos equipamentos e do ambiente, e o acondicionamento incorreto dos produtos prontos para o consumo, sem o controle de tempo/ temperatura, pode ocasionar uma contaminação por microrganismos patogênicos, como Staphylococcus aureus, Salmonella spp. e Escherichia coli (SOUZA et al., 2015).

Em relação à qualidade sanitária de um alimento, os coliformes fecais (E. coli) são indicadores de condições higiênico-sanitárias precárias, tanto da conservação, como do preparo desses alimentos. Outra preocupação é a presença de Salmonella neste tipo de alimento, principalmente porque o homem é uns dos principais portadores e transmissores dessa bactéria, causando surtos alimentares em diversos países (PINHEIRO et al., 2006; LUCENA et al., 2015).

O S. aureus é outro microrganismo que indica níveis de higiene nos alimentos. Essas bactérias são capazes de formar enterotoxinas, e se ingeridas pelo homem, podem provocar intoxicações alimentares que geram um grande desconforto ao indivíduo (SANTOS et al., 2012; MONTANARI et al., 2015).
Esse perigo na qualidade do sushi é por não haver tratamento térmico, portanto não ocorre a esterilização do alimento, deixando -o vulnerável à ação desses microorganismos patogênicos (CORREIA; RONCADA, 1997; MORAES; DARLEI; TIMM, 2019).

A ANVISA (Agência Nacional de Vigilância Sanitária), por meio da Resolução ${ }^{\circ}$ 216/04, estabeleceu critérios de boas práticas para serviços de alimentação, pelo fato da maioria das infecções parasitárias serem adquiridas pela transmissão oral-fecal, principalmente pela ingestão de água e alimentos contaminados. Além disso, a resolução exige que os funcionários que manipulam alimentos crus, devem, antes de manusear os alimentos, realizar a lavagem e a antisepsia das mãos, e adotar todas as medidas higiênicas para minimizar ao máximo o risco de contaminação cruzada (BRASIL, 2004; LODO et al., 2010; SILVA et al., 2018).

Embora a diarreia seja o sintoma mais comum de uma DTA, várias outras consequências podem ocorrer, como dores abdominais, problemas nos rins e fígado, desordens neurológicas e até a morte, principalmente com grupos mais vulneráveis, como crianças, idosos e grávidas (SCHLUNDT, 2002; MOUTA et al., 2014).

Contudo, a saúde e a higiene dos manipuladores de alimentos são extremamente fundamentais para garantir a qualidade das preparações e garantir um alimento seguro para o consumidor (SANTOS et al., 2012). Desta forma a escolha de fornecedores que garantam a qualidade do seu produto e que suas condições de estocagem e processamento estejam adequados é muito importante (MONTANARI et al., 2015). Diante do exposto, o objetivo deste estudo foi avaliar a qualidade microbiológica de sushi coletado em estabelecimento especializado e não especializado da cidade de Erechim, no Rio Grande do Sul. 


\section{Material e Métodos}

A pesquisa foi realizada utilizando-se o método de cunho experimental, de caráter exploratório, qualitativo e quantitativo.

Em relação aos materiais, todos os reagentes utilizados foram da marca Merck. A autoclave utilizada era da marca Phoenix, modelo AV75, a estufa utilizada era da marca Tecnal, modelo TE 393/2, e a balança utilizada era da marca Marte, modelo semi analítica AD.

\section{Coleta das amostras}

$\mathrm{Na}$ presente pesquisa de campo, o alimento escolhido para realizar as análises microbiológicas foi o sushi. A coleta foi realizada em 2 estabelecimentos da cidade de Erechim - RS, como consumidor anônimo e escolhidos por conveniência, identificados como estabelecimento "E" (especializado) e "NE" (não especializado). Foram realizadas 3 coletas de cada estabelecimento em um período de 10 dias cada $(1,2,3$, respectivamente), para melhor confiabilidade dos resultados encontrados, sendo coletado uma amostra de cada estabelecimento em cada período, totalizando 6 amostras, realizadas no mês de Janeiro de 2020.

Após cada coleta, as amostras foram acondicionadas em bolsa térmica com gelo, em suas embalagens originais e encaminhadas para o laboratório de Biotecnologia de Alimentos da Universidade Regional Integrada do Alto Uruguai e das Missões, Campus I URI Erechim, para a realização das análises microbiológicas, não excedendo 20 min entre a compra e o início das análises.

\section{Preparo das amostras}

A amostra do estabelecimento "E" era composta por arroz japonês, salmão, cream cheese e enrolado por alga marinha. A amos- tra do estabelecimento "NE" era composta por arroz japonês, vinagre, salmão, creme de queijo, gergelim torrado, gengibre, açúcar, sal e enrolado por alga marinha. As amostras foram cortadas, homogeneizadas e pesadas, utilizando as gramas necessárias para cada teste microbiológico.

\section{Análises microbiológicas}

Para estabelecer os padrões microbiológicos sanitários para alimentos, utilizou-se a resolução vigente $\mathrm{RDC}^{\circ} 12$, de 02 de janeiro de 2001 da Agência Nacional de Vigilância Sanitária (ANVISA), grupo $\mathrm{n}^{\circ} 22$ - item $\mathrm{B}$, que se titula como pratos prontos para $\mathrm{o}$ consumo (alimentos prontos de cozinhas, restaurantes e similares), enquadrando-se os alimentos a base de carnes, pescados e similares crus (quibe cru, carpaccio, sushi, sashimi, etc.) (BRASIL, 2001). Esta resolução define a tolerância de microrganismos para estes alimentos, exigindo as análises de Coliformes a $45^{\circ} \mathrm{C}$, Salmonella e $S$. aureus para sushi. No total foram realizadas 18 análises microbiológicas, sendo 9 análises para cada estabelecimento.

\section{Determinação de Coliformes a $45^{\circ} \mathrm{C}$}

Também conhecidas como coliformes fecais, essas bactérias são capazes de fermentar a lactose produzindo gás a $45^{\circ} \mathrm{C}$. Dentro deste grupo, a $E$. coli é a principal bactéria dos coliformes fecais (SILVA; JUNQUEIRA; SILVEIRA, 1997). Para a determinação de coliformes fecais, foi utilizada a técnica do Número Mais Provável (NMP), seguindo o Manual de Métodos de Análise Microbiológica de Alimentos (SILVA; JUNQUEIRA; SILVEIRA, 1997).

Esta análise foi realizada em 3 etapas diferentes, sendo elas preparação da amostra e diluições seriadas, teste presuntivo e teste confirmativo de coliformes fecais, descritas abaixo. 
Preparo da amostra e diluições: foi acesa uma lamparina entre uma balança e a amostra, para esterilizar o ambiente. Foram retiradas $25 \mathrm{~g}$ de cada amostra, utilizando faca e colher esterilizadas, e foram transferidas para um frasco erlenmeyer previamente esterilizado, contendo $225 \mathrm{~mL}$ de água peptonada $0,1 \%$ e, em seguida, foi homogeneizado (diluição $\left.10^{-1}\right)$. Logo após foi transferido $1 \mathrm{~mL}$ desta primeira diluição para um tubo de ensaio contendo $9 \mathrm{~mL}$ de água peptonada $0,1 \%$ e foi homogeneizado (diluição $10^{-2}$ ). Então, foi retirado $1 \mathrm{~mL}$ deste tubo de ensaio e adicionou-se em outro tubo de ensaio contendo, novamente, $9 \mathrm{~mL}$ de água peptonada $0,1 \% \mathrm{e}$ homogeneizou-se (diluição $10^{-3}$ ).

Teste presuntivo: nesta etapa foram retiradas alíquotas de $1 \mathrm{~mL}$ de cada diluição e inoculadas em 3 tubos contendo Caldo Lauril Sulfato Triptose (LST) com um tubo de Durham dentro. No total foram utilizados 9 tubos para cada amostra coletada, e em seguida, foram incubados a $35^{\circ} \mathrm{C}$ por $24 \mathrm{~h}$. Após esse período foi observado se ocorreu ou não a formação de gás dentro do tubo de Durham e/ou turvação do meio dentro do tubo. Ocorrendo a confirmação da presença de gás e/ou turvação, deve-se realizar o teste confirmativo para E. coli.

Teste confirmativo: para a confirmação da presença de Coliformes fecais, foi transferido 1 alçada, utilizando a alça de platina, para cada tubo positivo com caldo LST para igual quantidade de tubos contendo caldo EC (E. coli). Em seguida, foram incubados à $43^{\circ} \mathrm{C}$ por $24 \mathrm{~h}$. A presença de gás no interior dos tubos de Durham e/ou a turvação do meio é considerada reação positiva para contaminação de origem fecal.

\section{Determinação de Salmonella}

Seguindo a metodologia do Manual de Métodos de Análise Microbiológica de Alimentos (SILVA; JUNQUEIRA; SILVEIRA,
1997), a análise para determinação de Salmonella foi realizada em 3 etapas diferentes, sendo elas: Pré-enriquecimento em caldo não seletivo, Enriquecimento em caldo seletivo e Plaqueamento diferencial. Os resultados são classificados como ausência ou presença de Salmonella. As etapas estão descritas abaixo.

\section{Pré-enriquecimento em caldo não sele-} tivo: foi pesado, homogeneizado e transferido $25 \mathrm{~g}$ de cada amostra para um tubo erlenmeyer contendo $225 \mathrm{~mL}$ de caldo lactosado. Em seguida, foram incubados em estufa a $35^{\circ} \mathrm{C}$ por $24 \mathrm{~h}$.

Enriquecimento em caldo seletivo: esta fase objetiva inibir a multiplicação de outras bactérias e promove o crescimento preferencial de células de Salmonella (SILVA; JUNQUEIRA; SILVEIRA, 1997). Depois de esperado o tempo da etapa anterior, retirouse o caldo de pré-enriquecimento da estufa e foram transferidos $1 \mathrm{~mL}$ do caldo para um tubo de ensaio contendo $10 \mathrm{~mL}$ de caldo Tetrationato (TT) e $1 \mathrm{~mL}$ para outro tubo de ensaio contendo $10 \mathrm{~mL}$ de caldo Selenito Cistina (SC), para cada amostra. Então, foram novamente incubados em estufa a $35^{\circ} \mathrm{C}$ por $24 \mathrm{~h}$.

Plaqueamento diferencial: após o tempo estipulado de $24 \mathrm{~h}$, foram transferidos uma alçada do caldo TT e uma alçada do caldo SC para placas contendo o meio Rambach agar, e foram incubadas invertidas na estufa a $35^{\circ} \mathrm{C}$, por $24 \mathrm{~h}$. Após esse tempo observouse se houve desenvolvimento de colônias de Salmonella. Considera-se positiva a presença de Salmonella se houver crescimento de colônias cor de rosa escuro na placa.

Provas bioquímicas: uma vez selecionadas colônias típicas (positivas) nos meios indicadores seletivos, estas foram transferidas para meios de triagem: Ágar ferro triplo açúcar, Ágar TSI, Ágar Lisina Ferro - LIA, Ágar Motilidade-Indol-Ornitina - MIO (BRASIL, 2011). 


\section{Determinação de Staphylococcus aureus}

A contagem de $S$. aureus foi realizada conforme a metodologia seguindo a ISO 6888 - 1:1999, através do teste presuntivo - Unidade Formadora de Colônia (UFC/g).

Teste presuntivo: primeiramente, foram pesados e homogeneizados $25 \mathrm{~g}$ das amostras e transferidos para um erlenmeyer contendo $225 \mathrm{~mL}$ de água peptonada $0,1 \%$ (diluição $10^{-1}$ ). Depois foi repassado $1 \mathrm{~mL}$ dessa primeira diluição para um tubo de ensaio contendo $9 \mathrm{~mL}$ de água peptonada $0,1 \%$ (diluição $10^{-2}$ ). Em seguida, foi realizado o plaqueamento em superfície, repassando $0,1 \mathrm{~mL}$ de cada diluição $\left(10^{-1}\right.$ e $\left.10^{-2}\right)$ para suas respectivas placas de petri previamente esterilizadas em autoclave com o meio Ágar Baird-parker, espalhando cuidadosamente com uma alça de Drigalski. Em seguida, as placas foram incubadas invertidas em estufa a $35^{\circ} \mathrm{C}$ por $48 \mathrm{~h}$. Considera-se resultado positivo quando apresentar colônias circulares, pretas, pequenas, lisas, convexas, com bordas perfeitas ou massa de células esbranquiçadas nas bordas (ISO, 1999).

\section{Tratamento dos Dados}

Os resultados obtidos a partir das análises microbiológicas foram tabulados utilizandose ferramentas do BrOffice.org.

\section{Resultados e Discussão}

Os resultados das análises microbiológicas de coliformes fecais, Staphylococcus coagulase positiva e Salmonella realizadas nas amostras de Sushi comercializadas nos estabelecimentos especializados em culinária japonesa (E) e não especializados em culinária japonesa (NE) encontram-se na Tabela I.

Conforme a Tabela I, nos resultados de Escherichia coli do estabelecimento E, as amostras da coleta 1 e 3 apresentaram o valor máximo de contaminação, muito acima do limite permitido pela legislação, e assim, impróprias para o consumo. A amostra da coleta 2 encontrou-se com valor abaixo do limite $\left(9,3 \times 10^{1} \mathrm{NMP} / \mathrm{g}\right)$, porém, quase ao limite do aceito pela $\mathrm{RDC} \mathrm{n}^{\circ} 12$ de 2001 (BRASIL, 2001), que é $\leq 1,0 \times 10^{2} \mathrm{NMP} / \mathrm{g}$, estando portanto adequada aos padrões legais

Tabela I - Resultados das análises microbiológicas de coliformes fecais, Staphylococcus coagulase positiva e Salmonella realizadas nas amostras de Sushi do estabelecimento especializado (E) e não especializado (NE)

\begin{tabular}{ccccc}
\hline Análise & Referência & \multicolumn{3}{c}{ Estabelecimento E } \\
\cline { 3 - 5 } Microbiológica & RDC 12/2001 & Coleta 1 & Coleta 2 & Coleta 3 \\
\cline { 3 - 5 } & $\leq 1,0 \times 10^{2}$ & $\geq 2,4 \times 10^{2}$ & $9,3 \times 10^{1}$ & $\geq 2,4 \times 10^{2}$ \\
E. coli $(\mathrm{NMP} / \mathrm{g})$ & $\leq 5 \times 10^{3}$ & 0 & 0 & 0 \\
S. aureus $(\mathrm{UFC} / \mathrm{g})$ & Ausência & Ausência & Ausência & Ausência \\
\hline & & & Estabelecimento NE & \\
\hline E. coli $(\mathrm{NMP} / \mathrm{g})$ & $\leq 1,0 \times 10^{2}$ & $<3$ & $4,3 \times 10^{1}$ & $<3$ \\
S. aureus $(\mathrm{UFC} / \mathrm{g})$ & $\leq 5 \times 10^{3}$ & 0 & 0 & $4 \times 10^{1}$ \\
Salmonella & Ausência & Ausência & Ausência & Ausência \\
\hline
\end{tabular}


vigentes. Nas análises de Staphylococcus coagulase positiva, em nenhuma das amostras houve crescimento de colônias dessa bactéria, estando assim, de acordo com a legislação. Nas análises de Salmonella, todas as coletas $(1,2$ e 3$)$ apresentaram ausência desse microrganismo.

As análises microbiológicas do Estabelecimento NE demonstraram que este estabelecimento apresentou, para as 3 coletas (1, 2 e 3), resultados dentro dos limites estabelecidos pela RDC n ${ }^{\circ} 12$ de 2001 (BRASIL, 2001) para coliformes fecais, Staphylococcus coagulase positivo e Salmonella. Nas análises de coliformes fecais, a coleta 2 obteve o maior valor, 4,3 x $10^{1} \mathrm{NMP} / \mathrm{g}$, e mesmo assim abaixo do limite estabelecido pela legislação, que é de $\leq 1,0 \times 10^{2} \mathrm{NMP} / \mathrm{g}$. Referente às análises de Staphylococcus coagulase positivo, somente a coleta 3 apresentou contaminação desta bactéria $\left(4,0 \times 10^{1} \mathrm{UFC} / \mathrm{g}\right)$, porém, mesmo assim esteve com valores que atendem a legislação, estando apta para o consumo humano. Nas análises de Salmonella, todas as coletas apresentaram ausência desse microrganismo.

A contaminação por coliformes fecais ( $E$. coli) pode acontecer de diversas formas. A utilização de adornos por manipuladores de alimentos são fatores de risco para a contaminação, pois eles dificultam a higienização das mãos, unhas compridas possuem muitos microrganismos patógenos em caso de má higienização, uniformes em condições inadequadas de higiene também podem se tornar transmissores de bactérias, entre outros (ABREU; MEDEIROS; SANTOS, 2011).

O resultado encontrado nesta pesquisa para coliformes fecais em estabelecimento não específico foi diferente do encontrado na pesquisa de Mouta et al. (2014), que avaliaram três estabelecimentos não específicos em culinária japonesa e todas as amostras obtiveram resultados acima do permitido pela legislação. O mesmo estudo também avaliou um estabelecimento específico em culinária japonesa, que também apresentou resultados de contaminação fecal, este, igual ao encontrado na presente pesquisa.

Resultados similares foram encontrados em Aracaju-SE, para coliformes fecais, onde 28 de 35 amostras de sushi (80\%) comercializados em restaurantes estavam impróprias para o consumo, apresentando contagem de coliformes termotolerantes acima do permitido pela legislação brasileira (SANTOS et al., 2012).

Dreckmann et al. (2016), em seu estudo, avaliaram presença de coliformes termotolerantes em 10 amostras de sushi de 5 restaurantes especializados em culinária oriental, na cidade de Balneário Camboriú-SC, no qual todas as amostras apresentaram valores dentro do permitido pela legislação, diferente do encontrado pela presente pesquisa, na qual duas das três amostras do restaurante especializado apresentaram contaminação fecal.

No estudo de Moraes, Darley e Timm (2019), similar ao aqui realizado, em amostras de sushi comercializados em 5 restaurantes especializados em comida japonesa na cidade de Pelotas - RS, foi constatado que $50 \%$ das 10 amostras apresentaram coliformes fecais com valores acima do limite permitido pela ANVISA (2001).

Guimarães, Silva e Guimarães (2016), analisaram coliformes fecais em 30 amostras de sushi de estabelecimentos variados, entre supermercados e restaurantes nas cidades de Crato e Juazeiro do Norte, no Ceará, entre os meses de agosto e setembro de 2015, e verificaram que $100 \%$ das amostras estavam de acordo com o padrão preconizado pela resolução.

No geral, as amostras desta pesquisa para as análises de E. coli, 66,67\% estavam adequadas aos padrões estabelecidos pela legislação e 33,33\% estavam inadequadas.

O $S$. aures é um microrganismo que faz parte da flora natural do ser humano, 
e se encontra principalmente na pele e na região nasal das pessoas (SALES; SILVA, 2012). Neste estudo, somente a coleta 3 do estabelecimento NE mostrou pequena contaminação desta bactéria, demostrando que ocorreu maus hábitos de higiene por parte do manipulador. Em grandes quantidades, S. aureus pode se tornar patogênico e causar diversos tipos de infecções graves, como bacteremia, pneumonia, osteomielite, endocardite, miocardite, pericardite, meningite, abcessos musculares e cerebrais, e além disso, podem disseminar para outros tecidos com a geração de focos metastáticos (SALES e SILVA, 2012).

Dreckmann et al. (2016) coletaram 10 amostras de sushi de 5 restaurantes especializados em culinária oriental, na cidade de Balneário Camboriú -SC, e avaliaram que 4 restaurantes não evidenciam crescimento de colônias de $S$. aureus, sendo que apenas em 1 restaurante foi identificada presença dessa bactéria nas duas amostras coletadas, porém dentro dos valores de referência estabelecidos pela legislação brasileira.

Comparando a presente pesquisa com o estudo de Moraes, Darley e Timm (2019), nas 10 amostras de sushi comercializadas em 5 restaurantes especializados em comida japonesa, na cidade de Pelotas-RS, apenas 1 amostra (10\%) apresentou presença de $S$. aureus acima do permitido pela legislação. Os mesmos resultados foram encontrados no estudo de Souza et al. (2015), na qual das 15 amostras coletadas em 5 restaurantes especializados em culinária japonesa na cidade de João Pessoa - PB, entre julho e setembro de 2014, apenas 1 amostra apresentou $S$. aureus acima do valor de referência.

$\mathrm{Na}$ presente pesquisa, em nenhuma das amostras dos dois estabelecimentos (especializado e não especializado) houve crescimento de colônias de Staphylococcus aureus que ultrapassou o limite estabelecido pela RDC n ${ }^{\circ} 12$ (BRASIL, 2001). O mesmo resultado foi encontrado na pesquisa de Sato (2013), que avaliou 15 amostras de suhsi de estabelecimentos especializados e 15 amostras de estabelecimentos não especializados, onde todas as amostras tiveram crescimento de colônias de $S$. aureus, porém nenhuma ficou acima do limite da legislação brasileira, estando todas aptas para o consumo humano.

A contaminação do pescado por Salmonella acontece devido ao contato com as fezes de animais silvestres, domésticos e aves que circulam os tanques escavados na terra ou os reservatórios de água desprotegidos, o recebimento de esgotos ou processos de lixiviação que carregam os contaminantes do solo (SANTOS, 2015; GAZAL et al., 2018). A contaminação também pode ocorrer pela falta de higiene do estabelecimento, ou pelo estado sanitário inadequado dos manipuladores que, se adoeceram por salmonelose, são portadores do germe mesmo depois de terem desaparecido os sintomas da doença. Esses portadores excretam as bactérias do Gênero Salmonella pelas fezes, e na falta de higiene suficiente nas mãos, podem transmitir para os alimentos (MATACA, 2014).

Nenhum estabelecimento desta pesquisa apresentou crescimento de bactérias de $\mathrm{Sal}$ monella, e este resultado pode ser observado em pesquisas similares.

Já na pesquisa de Lucena et al. (2015), das 30 amostras coletadas de sushi nas cidades de João Pessoa e Campina Grande - PB, no período de julho a setembro de 2014, somente em 4 amostras foram confirmadas o crescimento de Salmonella, indicando falha no processo de Boas Práticas de Manipulação e falta de higiene dos manipuladores de alimentos, segundo os autores.

Resultados iguais foram encontrados no estudo de Santos et al. (2012), realizado em Aracaju - SE, entre agosto e novembro de 2010, no qual coletaram 35 amostras de sushi 
em 7 restaurantes especializados em gastronomia japonesa e em nenhuma das amostras foi identificada a presença de microrganismos do gênero Salmonella.

\section{Considerações Finais}

Diante dos resultados apresentados neste estudo, constatou-se que o estabelecimento E apresentou contaminação máxima por $E s$ chechiria coli (contaminação fecal) em 2 das 3 amostras analisadas, indicando que o alimento estava impróprio para o consumo. Este estabelecimento é especializado em culinária japonesa, isto é, só produz este tipo de alimento, portanto, é necessário maior cuidado com a higiene do local e dos manipuladores, pois sendo especializado, deveria garantir boa qualidade sanitária para seus clientes.

Neste estudo podemos observar que a população se encontra exposta a riscos de adquirirem uma DTA. Dessa forma, para solucionar ou minimizar o problema, é necessário uma implantação de programas de educação em saúde para estes tipos de estabelecimentos, como a implantação do Manual de Boas Práticas e os POPs (Procedimentos Operacionais Padronizados). Além disso, se faz necessário, também, treinamentos sobre educação sanitária para os manipuladores de alimentos e conscientização da importância da higiene em relação à segurança dos alimentos para, desta, forma buscar alimentos seguros, valorizando a saúde da população.

\section{REFERÊNCIAS}

BRASIL. Ministério da Saúde. Agência Nacional de Vigilância Sanitária. Resolução nº 12, de 02 de janeiro de 2001. Regulamento Técnico sobre padrões microbiológicos para alimentos.

BRASIL. Ministério da Saúde. Agência Nacional de Vigilância Sanitária. Resolução - RDC n 216, de 15 de setembro de 2004. Dispõe sobre Regulamento Técnico de Boas Práticas para Serviços de Alimentação.

BRASIL. Ministério da Saúde. Secretaria de Vigilância em Saúde. Manual técnico de diagnóstico laboratorial de Salmonella spp.: diagnóstico laboratorial do gênero Salmonella, Fundação Oswaldo Cruz. Laboratório de Referência Nacional de Enteroinfecções Bacterianas, Instituto Adolfo Lutz. Brasília, 2011, 60 p.

CORREIA, M.; RONCADA, M. J. Características microscópicas de queijos prato e mussarela e mineiro comercializados em feiras livres da cidade de São Paulo. Revista de Saúde Pública, v. 31, n. 3, p. 296-301, 1997.

DRECKMANN, M. V.; HINTZ, G. F. M.; LEMOS, M. P.; BRAMORSKI, A.; SCHMELING, T. B.; FELIPE, M. R. Qualidade higienicossanitária de sushis e sashimis comercializados em restaurantes orientais de Balneário Camboriú, SC. Revista Higiene Alimentar, v. 30, n. 252-253, p. 131-136, 2016.

GAZAL, L. E. S.; BRITO, K. C. T.; CAVALLI, L. S.; KOBAYASHI, R. K. T.; NAKAZATO, G.; OTUTUMI, L. K.; CUNHA, A. C.; NETO, J. A. S. P.; BRITO, B. G. Salmonella sp. em peixes qual a importância para sanidade em pescado? Pesquisa Agropecuária Gaúcha, v. 24, p. 55-64, 2018. 
GUIMARÃES, K. P.; SILVA, R. M. R.; GUIMARÃES, K. P. Investigação da qualidade microbiológica de sushis comercializados nas cidades de Crato e Juazeiro do Norte - CE. Revista e-ciência, v.4, n.2, p. 20-25, 2016.

ISO (1999) International Organization for Stardardization. Genebra. Suíça.

LODO, M.; OLIVEIRA, C. G. B.; FONSECA, A. L. A.; CAPUTTO, L. Z.; PACKER, M. L. T.; VALENTI, V. E.; FONSECA, F. L. A. Prevalência de enteroparasitas em município do interior paulista. Revista Brasileira de Crescimento e Desenvolvimento Humano, v. 20, n. 3, p. 769-777, 2010.

LUCENA, T. P.; SILVA FILHO, C. R. M.; ARAÚJO, C. D. L.; MORAIS, W. S. Ocorrência de coliformes termotolerantes e Salmonella spp. em sushis comercializados nas cidades de João Pessoa e Campina Grande / PB. $5^{\circ}$ Simpósio de Segurança Alimentar: Alimentação e Saúde. In: Anais [...] Bento Gonçalves, RS, 2015. Disponível em: http://www.ufrgs.br/sbctars-eventos/ssa5/anais/ficha. php. Acesso em: 10 jan. 2021.

LUNKES, L. C.; PAIVA, I. M.; RUBIM, F. M.; RIBEIRO, A. O.; MURGAS, L. D. S. Consumo de carnes e percepção dos universitários de Lavras-MG em relação a carne de peixe e seus benefícios à saúde. ARCHIVOS LATINOAMERICANOS DE NUTRICIÓN. Órgano Oficial de la Sociedad Latinoamericana de Nutrición, v. 68, n. 4, p. 295-302, 2018.

MATACA, A. R. Estudo da frequência de Salmonella spp. no pescado comercializado no Brasil. Dissertação (mestrado) - Universidade Federal de Minas Gerais, Escola de Veterinária Escola de Veterinária, UFMG, Belo Horizonte, 2014.

MEDEIROS, M. G. G. A.; CARVALHO, L. R.; FRANCO, R. M. Percepção sobre a higiene dos manipuladores de alimentos e perfil microbiológico em restaurante universitário. Revista Ciência e Saúde Coletiva, v. 22, n. 2, p. 383-392, 2017.

MONTANARI, A. S.; ROMÃO, N. F.; SOBRAL, F. O. S.; MARMITT, B. G.; SILVA, F. P. S.; MARTINELLI, T. C. A. Avaliação da qualidade microbiológica de sashimis de salmão, preparados e comercializados em restaurantes japonês no município de Ji-Paraná - RO. South American Journal of Basic Education, Technical and Technological, v.2, n.1, p. 4-16, 2015.

MORAES, T. P.; DARLEY, F. M.; TIMM, C. D. Avaliação microbiológica de sushi e sashimi preparados em restaurantes especializados. Revista de Ciências Agroveterinárias, v. 18, n. 2, p. 254-257, 2019.

MOUTA, R. M. A.; MELO, M. B.; ARAÚJO, A. B.; AGUIAR, F. L. L.; FONTENELLE, R. O. S. Qualidade microbiológica do sushi comercializado na cidade de Sobral - CE. Revista da Universidade Vale do Rio Verde, v. 12, n. 2, p. 277-284, 2014.

MOZAFFARIAN, D.; WU, J. H. Y. (n-3) Fatty Acids and Cardiovascular Health: Are Effects of EPA and DHA Shared or Complementary? The Journal of Nutrition, 614S-25S, 2012.

OLIVEIRA, J. M. de. O peixe e a saúde: das recomendações para o consumo às possibilidades ambientais de atendê-lo. Segurança Alimentar e Nutricional, v. 20, p. 141-146, 2013.

PINHEIRO, H. M. C.; VIEIRA, R. H. S. F.; CARVALHO, F. C. T.; REIS, E. M. F.; SOUSA, O. V.; VIEIRA, G. H. F.; RODRIGUES, D. P. Salmonella spp. e coliformes termotolerantes em sushi e sashimi comercializados na cidade de Fortaleza, Ceará. Boletim Técnico Científico do CEPENE, v. 14, n. 1, p. 19-32, 2006.

SALES, L. M.; SILVA, T. M. Staphylococcus aureus meticilina resistente: um desafio para a saúde pública. Acta Biomedica Brasiliensia, v. 3, n. 1, p.13, 2012.

SANTOS, A. A.; SIMÕES, G. T. N.; CRUZ, M. M.; FERREIRA, N. S. S.; LIMA, R. T. C; TUNON, G. I. L. Avaliação da qualidade microbiológica de sushi comercializado em restaurante de Aracaju, Sergipe. Scientia Plena, v. 8, n. 3, 2012. 
SANTOS, R. R. D. Ocorrência, tipagem molecular e capacidade de colonização de amostras de Salmonella enterica em peixes nativos. Belo Horizonte: UFMG, 2015.

SARTORI, A. G. O.; AMANCIO, R. D. Pescado: importância nutricional e consumo no Brasil. Segurança Alimentar e Nutricional, v. 19, n. 2, p. 83-93, 2012.

SATO, R. A. Características microbiológicas de sushis adquiridos em estabelecimentos que comercializam comida japonesa. UNESP, São Paulo. 2013.

SCHLUNDT, J. New directions in foodborne disease prevention. International Journal of Food Microbiology, v. 78, p. 3-17, 2002.

SILVA, D. F.; LIMA, C. M. B. L.; SILVA, A. B.; CAVALCANTE, U. M. B.; FIQUEIREDO JUNIOR, F. S.; FREITAS, F. I. S. Análise coproparasitológica de manipuladores de alimentos em restaurantes especializados em gastronomia japonesa. Arquivos de Ciências da Saúde, v. 25, n. 1, p. 29-34, 2018.

SILVA, N.; JUNQUEIRA, V. C. A.; SILVEIRA, N. F. A. Manual de métodos de análise microbiológica de alimentos. São Paulo: Livraria Varela, 1997.

SOUZA, T. J. F. F.; SILVA, J. N.; SILVA FILHO, C. R. M.; SANTOS, J. G. Microrganismos de interesse sanitário em sushis. Revista Instituto Adolfo Lutz, v. 74, n. 3, p. 274-279, 2015. 
